\title{
A CHARACTERIZATION OF THE DEFINITENESS OF A HERMITIAN MATRIX
}

\author{
by YIK-HOI AU-YEUNG and TAI-KWOK YUEN
}

(Received 11 January, 1972; revised 11 June, 1973)

1. Introduction. We denote by $F$ the field $R$ of real numbers, the field $C$ of complex numbers or the skew-field $H$ of real quaternions, and by $F^{n}$ an $n$-dimensional left vector space over $F$. If $A$ is a matrix with elements in $F$, we denote by $A^{*}$ its conjugate transpose. In all three cases of $F$, an $n \times n$ matrix $A$ is said to be hermitian (unitary resp.) if $A=A^{*}\left(A A^{*}=\right.$ identity matrix resp.). An $n \times n$ hermitian matrix $A$ is said to be definite (semidefinite resp.) if $u A u^{*} v A v^{*}>0\left(u A u^{*} v A v^{*} \geqq 0\right.$ resp.) for all nonzero $u$ and $v$ in $F^{n}$. If $A$ and $B$ are $n \times n$ hermitian matrices, then we say that $A$ and $B$ can be diagonalized simultaneously into blocks of size less than or equal to $m$ (abbreviated to d.s. $\leqq m$ ) if there exists a nonsingular matrix $U$ with elements in $F$ such that $U A U^{*}=\operatorname{diag}\left\{A_{1}, \ldots, A_{k}\right\}$ and $U B U^{*}=\operatorname{diag}\left\{B_{1}, \ldots, B_{k}\right\}$, where, for each $i=1, \ldots, k, A_{i}$ and $B_{i}$ are of the same size and the size is $\leqq m$. In particular, if $m=1$, then we say $A$ and $B$ can be diagonalized simultaneously (abbreviated to d.s.).

The purpose of this note is to give a characterization of the definiteness of a hermitian matrix in terms of simultaneous diagonalization. (For other characterizations see, for example, [3].)

2. Characterization of the definiteness of a hermitian matrix. In the following we shall use $A, B$ and $X$ to denote $n \times n$ hermitian matrices. We now give a characterization of the definiteness of a hermitian matrix.

Theorem 1. Let $A \neq O$. Then $A$ is definite if and only if $A$ and $X$ can be diagonalized simultaneously for all $X$.

Proof. Since any hermitian matrix can be diagonalized by a unitary matrix (for $F=R$ or $C$, this is well known, for $F=H$, see [4] or [6]), if $A$ is definite, then $A$ and $X$ can be d.s. Conversely, if $A$ is not definite, then we may assume that

Now take

$$
A=\operatorname{diag}\left\{A_{1}, A_{2}\right\}, \text { where } A_{1}=\left(\begin{array}{rr}
1 & 0 \\
0 & -1
\end{array}\right) \text { or }\left(\begin{array}{ll}
1 & 0 \\
0 & 0
\end{array}\right) \text {. }
$$

$$
X=\operatorname{diag}\left\{X_{1}, O\right\}, \text { where } X_{1}=\left(\begin{array}{ll}
0 & 1 \\
1 & 0
\end{array}\right) .
$$

Then $A_{1}$ and $X_{1}$ cannot be d.s. and hence (see [1] or [2]) $A$ and $X$ cannot be d.s.

In order to give a characterization of the semidefiniteness of a hermitian matrix, we need the following lemmas.

LEMMA 1. Let

and

$$
A_{1}=\left(\begin{array}{lll}
1 & & \\
& -1 & \\
& & 0
\end{array}\right), \quad X_{1}=\left(\begin{array}{rrr}
2 & -2 & 1 \\
-2 & 2 & 1 \\
1 & 1 & 0
\end{array}\right)
$$




$$
A_{2}=\left(\begin{array}{lll}
1 & & \\
& 1 & \\
& & -1
\end{array}\right), \quad X_{2}=\left(\begin{array}{rrr}
1 & -1 & 1 \\
-1 & 3 & -2 \\
1 & -2 & 1
\end{array}\right) \text {. }
$$

Then $A_{i}$ and $X_{i}$ cannot be d.s. $\leqq 2$ for $i=1,2$.

Proof. Suppose that $A_{i}$ and $X_{i}$ can be d.s. $\leqq 2$ by a nonsingular matrix $U_{i}=\left(\begin{array}{l}u_{i} \\ v_{i} \\ w_{i}\end{array}\right)$, where $i=1$ or 2 , and $u_{i}, v_{i}, w_{i}$ form a basis of $F^{3}$. Then at least one of them, say $u_{i}$, possesses the property that

$$
u_{i} A_{i} v_{i}^{*}=u_{i} A_{i} w_{i}^{*}=u_{i} X_{i} v_{i}^{*}=u_{i} X_{i} w_{i}^{*}=0 .
$$

Hence the subspace $L\left\{u_{i} A_{i}, u_{i} X_{i}\right\}$ spanned by $u_{i} A_{i}$ and $u_{i} X_{i}$ is of dimension less than or equal to 1 and $v_{i}$ and $w_{i}$ are in the orthogonal complement of this subspace. But, by direct calculation, it can be easily seen that such a basis cannot exist. This completes the proof.

The following lemma is obvious.

Lemma 2. Let $A=\operatorname{diag}\left\{A_{1}, O\right\}$ and $B=\operatorname{diag}\left\{B_{1}, O\right\}$, where $A_{1}$ and $B_{1}$ are of the same size. If $A$ and $B$ can be d.s. $\leqq 2$, then so can $A_{1}$ and $B_{1}$.

LEMMA 3. Let $A=\operatorname{diag}\left\{A_{1}, A_{2}\right\}$ and $B=\operatorname{diag}\left\{B_{1}, O\right\}$, where $A_{1}$ and $B_{1}$ are of size $k$, and let $B_{1}$ be nonsingular. If $A$ and $B$ can be d.s. $\leqq 2$, then so can $A_{1}$ and $B_{1}$.

Proof. By Lemma 2, without loss of generality, we may assume that $A_{2}$ is nonsingular. Suppose that $A$ and $B$ can be d.s. $\leqq 2$ by the matrix $U$. Let $u_{i}(i=1, \ldots, n)$ be the $i$ th row of $U$. Then $\left\{u_{1}, \ldots, u_{n}\right\}$ is a basis of $F^{n}$. Now put $u_{i}=\left(x_{i}, y_{i}\right)$, where $x_{i} \in F^{k}$ and $y_{i} \in F^{n-k}$. Let $\left\{x_{i_{1}}, \ldots, x_{i_{k}}\right\}$ be a basis of $F^{k}$ and let $M$ be the $k \times k$ matrix with rows $x_{i_{1}}, \ldots, x_{i_{k}}$; then $M$ is nonsingular. We shall show that $A_{1}$ and $B_{1}$ are d.s. $\leqq 2$ by $M$.

Let $\left\{j_{1}, \ldots, j_{n-k}\right\}=\{1, \ldots, n\} \backslash\left\{i_{1}, \ldots, i_{k}\right\}$. We first show that $U$ can be chosen so that it shall have $x_{j_{t}}=O$ for $t=1, \ldots, n-k$. To prove this we consider the matrix $U B U^{*}$, noting that its $(j, h)$ th entry is $x_{j} B_{1} x_{h}^{*}$. From the "diagonalized $\leqq 2$ " form of $U B U^{*}$ we see that, for any particular $j_{t}$, either (i) $x_{j_{t}} B_{1} x_{h}^{*}=0$ for all $h \in\{1, \ldots, n\} \backslash\left\{j_{t}\right\}$ or (ii) there is an $l$, with $l=j_{t}-1$ or $l=j_{t}+1$, such that $x_{j_{t}} B_{1} x_{h}^{*}=0$ for all $h \in\{1, \ldots, n\} \backslash\left\{j_{t}, l\right\}$.

If (i) holds or if (ii) holds with $l \in\left\{j_{1}, \ldots, j_{n-k}\right\}$, then $x_{j_{t}} B_{1} x_{h}^{*}=0$ for all $h \in\left\{i_{1}, \ldots, i_{k}\right\}$ and hence, since $B_{1}$ is nonsingular and the $k$ columns $x_{i,}^{*}, \ldots, x_{i_{k}}^{*}$ are linearly independent, we have $x_{j_{t}}=O$.

If (ii) holds with $l \in\left\{i_{1}, \ldots, i_{k}\right\}$, then $x_{j_{t}} B_{1} x_{h}^{*}=0$ and $x_{l} B_{1} x_{h}^{*}=0$ for all $h \in\left\{i_{1}, \ldots, i_{k}\right\} \backslash\{l\}$ and hence, since $B_{1}$ is nonsingular and the $k-1$ columns $x_{h}^{*}$ with $h \in\left\{i_{1}, \ldots, i_{k}\right\} \backslash\{l\}$ are linearly independent, $x_{j_{t}}$ and $x_{i}$ are linearly dependent, so that there is a $\lambda \in F$ such that $x_{j_{t}}=\lambda x_{1}$. Let $\tilde{u}_{j_{t}}=u_{j_{t}}-\lambda u_{t}=\left(O, \tilde{y}_{j_{t}}\right)$. Then $A$ and $B$ are d.s. $\leqq 2$ by the matrix with rows $u_{1}, \ldots, u_{f_{t}-1}$, $\tilde{u}_{j_{t}}, u_{j_{t}+1}, \ldots, u_{n}$. This result, applied in turn to each of the rows $u_{j_{t}}$ of $U$ for which $x_{j_{t}} \neq O$, establishes the existence of a matrix, which we shall again call $U$, in which the rows $u_{J_{\varepsilon}}$ $(t=1, \ldots, n-k)$ all have $x_{j_{t}}=O$ and the rows $u_{i_{1}}, \ldots, u_{i_{k}}$ are as in the original matrix $U$, and which is such that $A$ and $B$ are d.s. $\leqq 2$ by $U$. 
We assume, then, that $U$ has each $u_{j_{t}}$ of the form $\left(O, y_{j_{t}}\right)$ and note that, since $u_{j_{1}}, \ldots, u_{j_{n}-k}$ are linearly independent, $\left\{y_{j_{1}}, \ldots, y_{j_{n-k}}\right\}$ is a basis of $F^{n-k}$.

We prove that $A_{1}$ and $B_{1}$ are d.s. $\leqq 2$ by $M$, by showing that, if $r \neq s$ and

then

$$
u_{i_{r}} A u_{i_{s}}^{*}=u_{i_{r}} B u_{i_{s}}^{*}=0 \text {, }
$$

$$
x_{i_{r}} A_{1} x_{i_{s}}^{*}=x_{i_{r}} B_{1} x_{i_{s}}^{*}=0 .
$$

For each $i_{r}$, we have two cases:

Case 1. $u_{i_{r}} A u_{j_{s}}^{*}=0$ for all $t=1, \ldots, n-k$.

In this case, we have

$$
y_{i_{r}} A_{2} y_{j_{t}}^{*}=0 \text { for all } t=1, \ldots, n-k \text {, }
$$

and evidently $y_{i_{r}}=O$ as $A_{2}$ is nonsingular. Therefore

$$
x_{i_{r}} A_{1} x_{i_{s}}^{*}=x_{i_{r}} B_{1} x_{i_{s}}^{*}=0 \text { if } u_{i_{r}} A u_{i_{s}}^{*}=u_{i_{r}} B u_{i_{s}}^{*}=0 \text {. }
$$

Case 2. There is some $j_{t}$ such that $u_{i_{r}} A u_{j_{t}}^{*} \neq 0$.

In this case $j_{t}$ is either $i_{r}-1$ or $i_{r}+1$, and the subspace spanned by $u_{i_{r}} A, u_{i_{r}} B, u_{j_{t}} A, u_{j_{t}} B$ is of dimension 2 with a basis $\left\{u_{i_{r}} B, u_{j_{t}} A\right\}$. Hence there exist $a$ and $b$ in $F$ such that

$$
u_{i,} A=a u_{i_{r}} B+b u_{j_{t}} A \text {, }
$$

and, by using the fact that $A_{2}$ is nonsingular, we have $y_{i_{r}}=b y_{j_{t}}$. If $u_{i_{r}} A u_{i_{s}}^{*}=u_{i_{r}} B u_{i_{s}}^{*}=0$, then

and hence

$$
u_{j_{t}} A u_{i_{s}}^{*}=u_{j_{t}} B u_{i_{s}}^{*}=0 \text { as } r \neq s \text {, }
$$

Consequently, we have

$$
\left(u_{i_{r}}-b u_{j_{\mathrm{r}}}\right) A u_{i_{s}}^{*}=\left(u_{i_{r}}-b u_{j_{r}}\right) B u_{i_{s}}^{*}=0
$$

$$
x_{i_{r}} A_{1} x_{i_{s}}^{*}=x_{i_{r}} B_{1} x_{i_{s}}^{*}=0 .
$$

THEOREM 2. (a) If $A$ is semidefinite, then $A$ and $X$ can be diagonalized simultaneously into blocks of size $\leqq 2$ for all $X$. (b) If $n \geqq 3$ and $A$ and $X$ can be diagonalized simultaneously into blocks of size $\leqq 2$ for all $X$, then $A$ is semidefinite.

Proof. (a) If $n \leqq 2$, then the first statement is obviously true. We now suppose it holds for all $k<n$ with $n>2$. Let $A$ be semidefinite and of size $n$. Then, without loss of generality, we may assume that

$$
A=\operatorname{diag}\left\{I_{m}, O\right\} \text { and } X=\left(\begin{array}{ll}
X_{11} & X_{12} \\
X_{12}^{*} & X_{22}
\end{array}\right),
$$

where $I_{m}$ is the $m \times m$ identity matrix $(0<m<n)$ and $X_{11}$ is of size $m$. If $X_{22} \neq 0$, then obviously we can reduce the problem to the case for $n-1$, and hence, by our assumption, statement (a) is true. We now assume that

$$
X=\left(\begin{array}{cc}
X_{11} & X_{12} \\
X_{12}^{*} & 0
\end{array}\right)
$$


with $X_{12} \neq O$. Let $v$ be a nonzero vector in $F^{n-m}$ such that $v X_{12}^{*} \neq O$, and let $u_{1}=(O, v)$ and $u_{2}=\left(v X_{12}^{*}, O\right)$ be vectors in $F^{n}$. Then

$$
u_{1} A=O, u_{1} X=u_{2} A=\left(v X_{12}^{*}, O\right) \text { and } u_{2} X=\left(v X_{12}^{*} X_{11}, v X_{12}^{*} X_{12}\right) \text {. }
$$

Since $v X_{12}^{*} \neq O$, we have $v X_{12}^{*} X_{12} v^{*}=v X_{12}^{*}\left(v X_{12}^{*}\right)^{*} \neq 0$. Hence $v X_{12}^{*} X_{12} \neq O$ and the vectors $\left(v X_{12}^{*}, O\right)$ and $\left(v X_{12}^{*} X_{11}, v X_{12}^{*} X_{12}\right)$ are linearly independent. Therefore the subspace $L$ spanned by $u_{1} A, u_{1} X, u_{2} A$ and $u_{2} X$ is of dimension 2 . If we denote by $L^{1}$ the orthogonal complement of $L$ and take a basis $\left\{u_{3}, \ldots, u_{n}\right\}$ in $L^{\perp}$, then it can easily be seen that $\left\{u_{1}, \ldots, u_{n}\right\}$ is a basis in $F^{n}$. Let $U$ be the matrix whose $i$ th row is $u_{i}(i=1, \ldots, n)$. Then $U$ is nonsingular with elements in $F$, and $U A U^{*}=\operatorname{diag}\left\{M_{1}, M_{2}\right\}, U X U^{*}=\operatorname{diag}\left\{N_{1}, N_{2}\right\}$, where $M_{1}$ and $N_{1}$ are $2 \times 2$ matrices and $M_{2}$ is semidefinite. By the induction assumption $M_{2}$ and $N_{2}$ can be d.s. $\leqq 2$, and therefore so can $A$ and $X$.

(b) If $n \geqq 3$ and $A$ is not semidefinite, then we may assume that

where

$$
A=\operatorname{diag}\left\{A_{1}, A_{2}\right\} \text {, }
$$

Take

$$
A_{1}=A_{1}^{(1)}=\left(\begin{array}{lll}
1 & & \\
& -1 & \\
& & 0
\end{array}\right) \text { or } A_{1}^{(2)}=\left(\begin{array}{lll}
1 & & \\
& 1 & \\
& & -1
\end{array}\right) \text {. }
$$

where

$$
X=\operatorname{diag}\left\{X_{1}, O\right\}
$$

$$
X_{1}=X_{1}^{(1)}=\left(\begin{array}{rrr}
2 & -2 & 1 \\
-2 & 2 & 1 \\
1 & 1 & 0
\end{array}\right) \quad \text { or } \quad X_{1}^{(2)}=\left(\begin{array}{rrr}
1 & -1 & 1 \\
-1 & 3 & -2 \\
1 & -2 & 1
\end{array}\right) \text {. }
$$

By Lemma $1, A_{1}^{(k)}$ and $X_{1}^{(k)}$ cannot be d.s. $\leqq 2$ for $k=1,2$. Hence by Lemma 3, $A$ and $X$ cannot be d.s. $\leqq 2$. Thus the theorem is proved.

Since it is obvious that, if $A$ and $B$ are semidefinite and of size 2, then $A$ and $B$ are d.s., the following theorem, which is already known (see [2] or [5]), follows immediately from Theorem 2, Part (a).

THEOREM 3. If $A$ and $B$ are semidefinite, then $A$ and $B$ can be diagonalized simultaneously.

\section{REFERENCES}

1. Y. H. Au-Yeung, A necessary and sufficient condition for simultaneous diagonalization of two hermitian matrices and its application, Glasgow Math. J. 11 (1970), 81-83.

2. Y. H. Au-Yeung, A note on some theorems on simultaneous diagonalization of two hermitian matrices, Proc. Cambridge Philos. Soc. 70 (1971), 383-386.

3. C. R. Johnson, Positive definite matrices, Amer. Math. Monthly 77 (1970), 259-264.

4. H. C. Lee, Eigenvalues and canonical forms of matrices with quaternion coefficients, Proc. Roy. Irish Acad. Sect. A 52 (1949), 253-260.

5. R. W. Newcomb, On the simultaneous diagonalization of two semi-definite matrices, Quart. Appl. Math. 19 (1961), 144-146. $1-14$.

6. J. Radon, Lineare Scharen orthogonaler Matrizen, Abh. Math. Sem. Univ. Hamburg 1 (1922),

UNIVERSITY OF HONG KONG 